\title{
Relação do custo de transporte da cana-de-açúcar em função da distância
}

Ratio of the cost of transportation of sugarcane due to the distance

\author{
Renato Frias Françoso; Aline Bigaton $2^{*}$; Haroldo José Torres da Silva ${ }^{3}$, Pedro \\ Valentim Marques ${ }^{4}$
}

\begin{abstract}
1 ESALQ/USP - Bacharel em Engenharia Agronômica - Rua Alexandre Herculano 120, sala B12, Vila Monteiro - CEP 13418-445 - Piracicaba (SP), Brasil

2 UNICAMP - Mestranda em Planejamento de Sistemas Energéticos (Faculdade de Engenharia Mecânica)

- Rua Alexandre Herculano 120, sala B12, Vila Monteiro - CEP 13418-445 - Piracicaba (SP), Brasil

3 ESALQ/USP - Doutorando em Economia Aplicada (Departamento de Economia, Administração e Sociologia) - Rua Alexandre Herculano 120, sala B12, Vila Monteiro - CEP 13418-445 - Piracicaba (SP), Brasil

${ }^{4}$ ESALQ/USP - Professor Titular (Departamento de Economia, Administração e Sociologia) - Rua Alexandre Herculano 120, sala T4, Vila Monteiro - CEP 13418-445 - Piracicaba (SP), Brasil
\end{abstract}

\section{Resumo}

Esta publicação apresenta os resultados do levantamento de custos de corte, carregamento e transporte da produção da cana-de-açúcar para a safra 2016/17 no estado de São Paulo. Os resultados apontaram uma grande disparidade de valores. Na região de Olímpia, o custo, por tonelada, referente a uma distância de $25 \mathrm{~km}$ é de $\mathrm{R} \$ 26,77$, enquanto que na região de Andradina o valor é de $\mathrm{R} \$ 37,25$. As variações dos dados podem estar relacionadas a diferentes variáveis, como o tipo de relevo, qualidade das estradas, preço do combustível na região, valorização da mão-de-obra, contratos particulares com usinas e prestadores de serviço, representando possibilidades de estudos futuros.

Palavras-chave: matéria-prima, raio médio, sucroenergético

\section{Abstract}

This publication presents the results of the survey of cutting, loading and transportation costs of sugarcane production for the 2016/17 year crop in the state of São Paulo. The results pointed to a large disparity of values. In the region of Olimpia, for example, the cost, per ton, for a distance of $25 \mathrm{~km}$ is $R \$ 26.77$, while in the region of Andradina the value is $R \$ 37.25$. The variation of the data might be related to different variables, such as the type of relief, road quality, fuel price in the region, valorization of the workforce, private contracts with mills and service providers, representing possibilities of future studies.

Keywords: raw material, mean radius, sugar-energy industry

\section{Introdução}

O Brasil é reconhecido como sendo o maior produtor de cana-de-açúcar do mundo, apresentando uma área plantada na safra 2015/16 em torno de 10.570.063 hectares, sendo o Sudeste a maior região produtora representando 59,31\% da área total (6.626.894 hectares) e 64,85\% da produção de cana-de-açúcar (475.752.411 t) no país (UDOP, 2016).

Segundo a UNICA (2016), o estado de São Paulo lidera a moagem de cana-deaçúcar no Brasil, com 55\% de toda moagem nacional, o equivalente a 368.322,65 mil toneladas de cana, enquanto que o segundo estado com maior produção (Goiás)

\footnotetext{
*Autor correspondente: <alinebigaton@pecege.com> Enviado: 17 jan. 2017

Aprovado: 08 fev. 2017
} 
aparece com apenas $11 \%$ de toda a produção (73.521,68 mil toneladas de cana), conforme ilustrado na Figura 1.

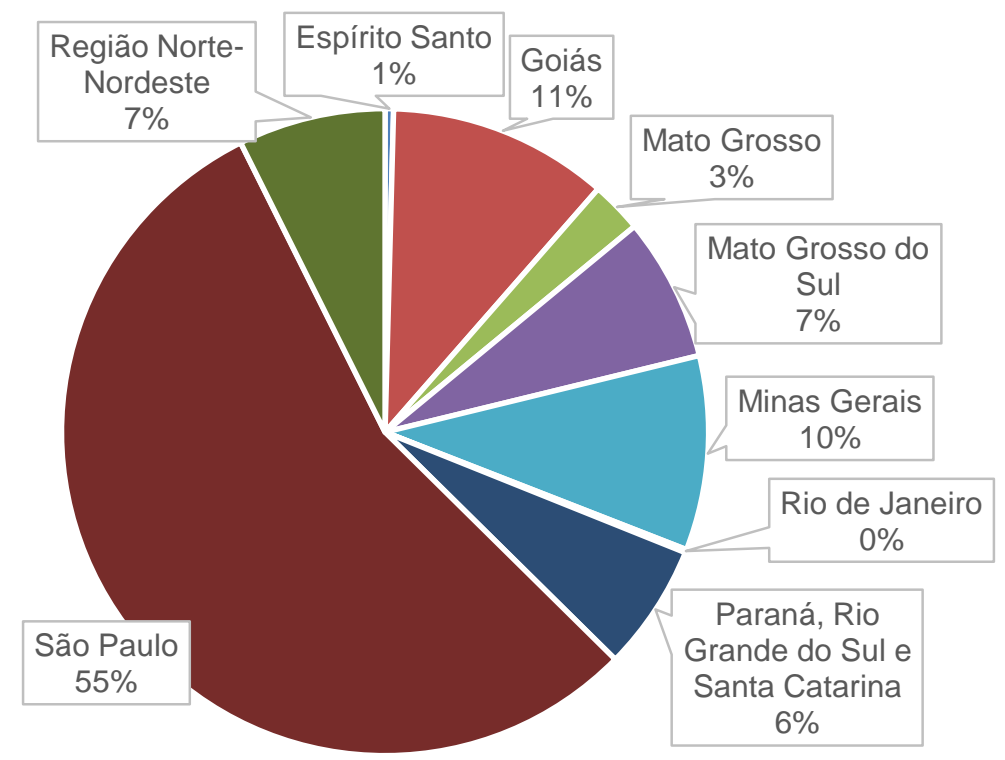

Figura 1. Moagem de cana no Brasil safra 2015/16 Fonte: UNICA (2016)

No sentido da importância que a cana-de-açúcar representa, destaca-se a etapa da colheita da matéria-prima, o principal componente do custo operacional de produção, correspondendo a cerca de $40 \%$ dos desembolsos na produção da cultura e esta, por sua vez, corresponde por $60 \%$ do custo de produção do açúcar e etanol (PECEGE, 2015). A operação de colheita está em plena evolução, migrando do sistema tradicional de colheita manual de cana inteira com queima prévia do canavial, para o sistema de colheita mecanizada de cana picada sem queima do canavial. A principal razão para esta transição está nas leis Federal e Estadual (para São Paulo) que estabelecem cronogramas para a redução e fim das queimadas nos canaviais. (NOVACANA, 2013).

A evolução da colheita de cana-de-açúcar no centro-sul do Brasil cresceu cerca de $60 \%$ desde 2000 até 2013 , mostrando que nessa região quase $90 \%$ da colheita é mecanizada (Belardo, 2015). Parte dessa evolução está relacionada ao transporte da cana, que cresceu bastante nos últimos anos, principalmente com a atuação do Centro de Tecnologia Canavieira [CTC] em parceria com fabricantes de carrocerias. O objetivo principal tem sido sempre reduzir os custos de transporte e se adaptar às mudanças no sistema de colheita. A distância média de transporte (do campo até as usinas) no 
Centro-Sul gira em torno de $25 \mathrm{~km}$ e tem aumentado nos últimos anos, acompanhando o contínuo aumento de escala das usinas (NOVACANA, 2013).

Considerando a importância do estado de São Paulo na produção de cana-deaçúcar, bem como o desenvolvimento da etapa agrícola da colheita ao longo dos últimos anos, foram levantados dados quanto ao custo de transporte da matéria-prima em diferentes regiões. A coleta desses dados ocorreu simultaneamente ao levantamento dos custos de produção de cana-de-açúcar de fornecedores, realizado pelo Programa de Educação Continuada em Economia e Gestão de Empresas [PECEGE], em parceria com a Confederação Nacional de Agricultura e Pecuária [CNA]. Segui-se a metodologia de painéis, que é caracterizada por encontros presenciais, em que os participantes, em geral produtores rurais e técnicos de cooperativas, associações e sindicatos, definem de forma consensual a unidade produtiva modal da região em questão, indicando os coeficientes técnicos e econômicos pré-determinados que definem o pacote tecnológico de produção de cana.

\section{Custo total de corte, transbordo e transportes [CTT] em razão da distância por região}

A partir de uma distância determinada de $25 \mathrm{~km}$, observou que há uma grande disparidade dos dados. Na região de Olímpia, o CTT, por tonelada, foi de $R \$ 26,77$, enquanto que na região de Andradina esse mesmo CTT apresentou um valor de $R \$$ 37,25. A mesma disparidade foi observada nos valores isolados de CTT. Considerando somente o valor do corte, o painel de Barra Bonita, apresentou custo, por tonelada, em torno de $R \$ 21,74$ e enquanto que Paulo de Faria o valor foi menor ( $R \$ 12,21)$. Em relação ao transbordo, o valor, por tonelada, flutua entre $\mathrm{R} \$ 4,94$ em Santa Bárbara D’Oeste e $R \$ 9,74$ em Valparaíso (Tabela 1 ).

De acordo com a Figura 2, na qual todos os dados foram agrupados em média, mínimo e máximo, quanto maior a distância, maior a disparidade entre os dados e maior a cobrança do transporte na CTT. Observou-se que há uma tendência de encontrar valores com menos disparidades no final da curva. Uma das hipóteses para esse acontecimento pode estar associada à quantidade de estradas em boas condições de rodagem, pelas quais o caminhão percorre. A função da linha de tendência dos pontos amostrados pode ser utilizada para determinar um valor aproximado do custo do transporte para qualquer distância requerida (Figura 2). 
Tabela 1. Custo de corte, transbordo e transporte de cana de açúcar em diferentes cidades do estado de São Paulo

\begin{tabular}{ccccccc}
\hline Safra & Painel & Distância & Transporte & Transbordo & Corte & Total \\
\hline & & $-------k m-------$ & $------------------R \$$ t $^{-1}-------------------$ \\
$16 / 17$ & Paulo de Faria & 25,00 & 7,54 & 7,57 & 12,21 & 27,32 \\
$16 / 17$ & Monte Aprazível & 25,00 & 7,10 & 4,98 & 16,02 & 28,10 \\
$16 / 17$ & Andradina & 25,00 & 11,59 & 6,33 & 19,33 & 37,25 \\
$16 / 17$ & Valparaiso & 25,00 & 7,49 & 9,74 & 15,75 & 32,98 \\
$16 / 17$ & Olímpia & 25,00 & 5,92 & 7,31 & 13,54 & 26,77 \\
$16 / 17$ & Guariba & 25,00 & 7,55 & 5,99 & 15,75 & 29,29 \\
$16 / 17$ & Barra Bonita & 25,00 & 7,42 & 7,07 & 21,74 & 36,23 \\
$16 / 17$ & Jaú & 25,00 & 7,42 & 5,99 & 15,75 & 29,16 \\
$16 / 17$ & Assis & 25,00 & 7,72 & 8,34 & 13,47 & 29,53 \\
$16 / 17$ & Piracicaba & 25,00 & 8,43 & 5,65 & 15,60 & 29,68 \\
$16 / 17$ & Ourinhos & 25,00 & 7,65 & 6,35 & 16,00 & 30,00 \\
$16 / 17$ & Chavantes & 25,00 & 6,49 & 6,05 & 15,90 & 28,44 \\
$16 / 17$ & Bariri & 25,00 & 9,44 & 7,31 & 14,95 & 31,70 \\
$16 / 17$ & Santa Barbara & 25,00 & 6,72 & 4,94 & 15,75 & 27,41 \\
$16 / 17$ & Novo Horizonte & 25,00 & 8,53 & 5,32 & 15,16 & 29,01 \\
$16 / 17$ & Igarapava & 25,00 & 7,00 & 5,65 & 14,86 & 27,51 \\
\hline
\end{tabular}

Fonte: Dados originais da pesquisa

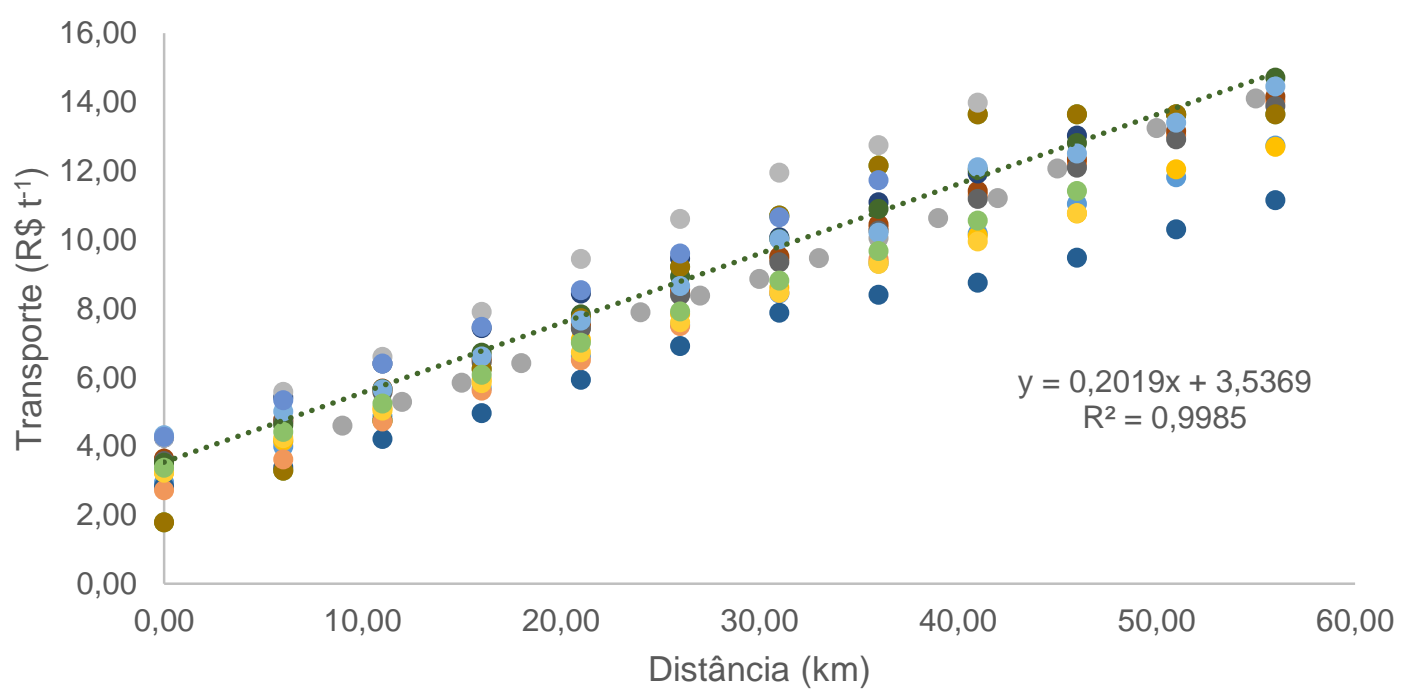

Figura 2. Dispersão dos dados amostrados em diferentes regiões do estado de São Paulo

Fonte: Dados originais da pesquisa

O custo médio, por tonelada, apurado na safra 2016/2017 para a operação de transbordo foi de $\mathrm{R} \$ 6,54$ e de $\mathrm{R} \$ 15,74$ para o corte (Tabela 2). No entanto, especificamente a operação de transporte, possui uma relação direta e positiva com a 
distância percorrida da propriedade até a usina. Assim como citado, para fins de balizamento, foi determinada uma distância média de $25 \mathrm{~km}$, a qual gerou um custo de transporte, por tonelada, na faixa de $\mathrm{R} \$ 7,75$, totalizando o custo total de CTT de $\mathrm{R} \$ 30,03$.

Tabela 2. Estatística descritiva dos dados de corte, transbordo e transporte [CTT] para a safra 2016/2017 no estado de São Paulo

\begin{tabular}{lccccc}
\hline & Unidade & Corte & Transbordo & Transporte & CTT \\
\hline Média & ${\mathrm{R} \$ \mathrm{t}^{-1}}^{-1}$ & 15,74 & 6,54 & 7,75 & 30,03 \\
Desvio Padrão & & 2,21 & 1,29 & 1,31 & 3,43 \\
$\mathrm{CV}$ & $\%$ & 14,04 & 19,74 & 16,99 & 11,73 \\
\hline
\end{tabular}

Fonte: Dados originais da pesquisa

Na distância mínima de transporte $(0 \mathrm{~km})$, observou-se que os pontos divergiram, por tonelada, por aproximadamente $\mathrm{R} \$ 2,00$, enquanto que no final da dispersão (valores acima de $40 \mathrm{~km}$ ) os pontos tenderam a aumentar sua diferença, chegando a aproximadamente $\mathrm{R} \$ 5,00$. De acordo com PECEGE/CNA (2015), para a safra 2015/2016, o custo da colheita, por tonelada, para uma distância média de $25 \mathrm{~km}$ foi de $R \$ 28,00$, ou seja, o custo de CTT na safra atual está $R \$ 2,03$ maior em relação à safra passada.

Os custos associados à distância média do canavial à usina desencadeiam algumas discussões quanto à quantidade ideal de cana a ser transportada do campo para a usina, a qual pode ser modificada de acordo com variações do ambiente, como clima, localização das frentes de corte (áreas muito distantes), tipo de estrada e especificações da frota. Por outro lado, a ociosidade de caminhões no pátio também é motivo de grande preocupação devido ao alto custo de investimentos, mão-de-obra e combustível, além da falta que estes veículos fazem no campo, pois se não houver caminhões disponíveis para receber a cana colhida, não haverá trabalho para operários e máquinas (Vian e Marin, 2009).

Além disso, é imprescindível estudos que possibilitem tomadas de decisão quanto à seleção do modelo e fabricante da colhedora, dimensionamento ideal da frente de colheita, manutenção dos equipamentos, planejamento da estrutura de apoio, bem como uma política de avaliação da renovação ou não das frotas. Tais práticas podem reduzir o custo fixo e garantir eficiência na operação de colheita.

\section{Conclusões}


Com a utilização da função de custo de transporte, pode-se extrair um valor aproximado do custo CTT e assim utilizar como ferramenta para determinar o valor aproximado a ser pago pela atividade.

Para o estado de São Paulo, a região de Paulo Faria apresenta um menor custo por tonelada de CTT, enquanto a região de Andradina apresenta maior custo, sendo uma diferença de aproximadamente $R \$ 10,00$, aproximando de $R \$ 800,00$ por hectare de diferença, onerando o valor da atividade e deixando-a menos atrativa.

Para estudos futuros, pode-se analisar as variações encontradas dos dados, que podem estar relacionadas a diferentes variáveis, como o tipo de relevo, qualidade das estradas, preço do combustível na região, valorização da mão-de-obra, contratos particulares com usinas e prestadores de serviço, ou seja, apresenta um leque bastante abrangente para pesquisa.

\section{Referências Bibliográficas}

Belardo, G.C.; Cassia, M.T.; Silva, R.P. 2015. Processos Agrícolas e Mecanização da Cana de Açúcar. SBEA. Jaboticabal, São Paulo, Brasil.

NOVACANA. 2013. Como é feito o transporte de cana-de-açúcar no Brasil. Disponível em: <https://www.novacana.com/cana-de-acucar/transporte-da-cana-brasil/>. Acesso em: 26 jun. 2016.

Programa de Educação Continuada em Economia e Gestão de Empresas [PECEGE]. 2015. Custo de produção de cana-de-açúcar, açúcar, etanol e bioeletricidade no Brasil: fechamento da safra 2014/15 e acompanhamento da safra 2015/16. Piracicaba: Universidade de São Paulo, Escola Superior de Agricultura "Luiz de Queiroz", Programa de Educação Continuada em Economia e Gestão de Empresas/Departamento de Economia, Administração e Sociologia. 2015. 73p. Relatório apresentado a Confederação da Agricultura e Pecuária do Brasil [CNA].

União dos Produtores de Bioenergia [UDOP]. 2016. Área plantada, área colhida e produção, por ano da safra e produto. Disponível em:

<http://www.udop.com.br/download/estatistica/area_cultivada/set2016_area_plantada_ colhida_prod_regioes.pdf>. Acesso em: 03 nov. 2016.

União da Indústria de Cana-de-Açúcar [UNICA]. 2016. Disponível em: <http://www.unicadata.com.br/>. Acesso em: 28 out. 2016.

Vian, C.E.F.; Marin, F.R. 2009. Logística e Transporte. In: Marin, F.R. Árvore do Conhecimento: Cana de açúcar. Ageitec. Campinas, SP, Brasil. Disponível em: < http://www.agencia.cnptia.embrapa.br/gestor/cana-deacucar/arvore/CONTAG01_133_22122006154842.html>. Acesso em: 20 nov. 2016. 